\title{
Giese, Rachel. (2018). Boys: What it Means to Become a Man. Toronto: HarperCollins Publishers Ltd.
}

\author{
Reviewed by: Nicolle-Ann Tamayo, MacEwan University
}

Rachel Giese explores boys' societal pressures and expectations in "Boys: What it Means to Become a Man." As the parent of a boy, she aims to understand his reality and the challenges he will likely encounter. Giese supports her analysis of modern masculinity with evidence from activists, educators, history, and social scientific research. Her introduction of the "Man Box" perpetuates throughout the book and displays the consequences of how society views boys and how boys understand themselves. Giese also discusses the role of toxic masculinity in mental health and friendships. Additionally, she explores how race shapes masculinity. Furthermore, she examines the role of toxic masculinity in sports and sex.

Giese examines Jeff Perera's metaphor of the "Man Box," which depicts rigid expectations and traits of masculinity for boys. In the box, Perera wrote stereotypes associated with manliness like, ". . . tough, brave, emotionless, heterosexual" (p. 1). Outside of the box were words that paralleled masculine stereotypes such as "pussy, batty boy, bitch and mama's boy" (p. 1). Perera's intention of the Man Box exercise was to indicate "the formula for manhood is the denial of everything perceived as soft ... or feminine" (p. 2). Even so, following the stereotypes of the Man Box is not enough to define masculinity. Masculinity is not monolithic; it is composed of race, class, ethnicity, nationality, and sexuality, resulting in boys aiming unattainable standards of manliness (p. 4). Understanding the nuances in definitions of masculinity and how they contrast 
with the Man Box explains why gay men and transgender individuals are frequently the targets of violence for challenging traditional masculinity (p. 4). Giese suggests the Man Box is an essential tool for sociologists to understand the behaviours and limitations boys experience throughout their socialization (p. 2).

Furthermore, Giese emphasizes how the ideals of masculinity from the Man Box affect mental health. Giese highlights that the men in the Man Box are more likely to "experience depression and think about suicide" and are "less likely to seek psychological and emotional help," fearing looking weak. (p. 14). Giese includes research from social worker Tyvon Hewitt; he concludes that men over eighteen felt disappointed if they did not live up to masculine expectations (p. 15). Hewitt emphasizes messages that perpetuate the "provider's complex" start at home, where parents enforce messages like "man up" and encourage boys to hide their emotions (p. 16). Encouraging men to hide how they feel conveys that their feelings are unimportant. Giese strategically includes Hewitt's findings to emphasize that internalizing toxic masculinity is frequently the result of messages from their primary socialization.

In addition to messages of masculinity from parents, pop culture also lays out expectations of masculinity that can become detrimental to boys' mental health. Boys try to identify and relate to characters that they watch on television. Superhero's popularity has introduced a standard of manliness that few "individuals can obtain" (p.152). Aiming to gain superheroes' physique, psychologists and doctors specify that the images of extreme male bodies correlate to an increase of eating disorders among boys and men (p. 152).

Due to the restrictions of the Man Box, lacking healthy relationships with themselves 
frequently prevents boys from developing deep connections in friendships. The phrase "no homo" safeguards boy friendships from being too "soft ... or feminine" (p. 2). Giese emphasizes historical male friendships were more valuable than marriages, and some relationships between male friends were sexual (p. 52). Homosexuality is a modern concept; it was only until the mid-1800's that cultural norms of sexual male friendships were labelled as homosexuality (p. 53). The introduction of homosexuality resulted in a cultural shift, viewing male love as suspicious and unnatural (p. 54). Giese emphasizes the stark line between male friendships and homosexual relationships creates distance between men, resulting in some male friendships withholding emotional closeness in fear of looking "gay" (p. 56). In contrast to the Man Box, emotional intimacy in male friendships is necessary because it increases happiness and alleviates loneliness (pp. 55-56).

Childhood boy friendships are significant because bonding with others makes them feel less lonely in their transition from childhood to adolescence (pp. 59-60). However, as they reach adolescence, they lose closeness from childhood as some feel less comfortable sharing emotions (p. 60). Professor of psychology Niobe Way indicates as boys become more aware of same-sex intimacy, they distance themselves from their friendships and turn to romantic relationships (p. 60). Giese incorporates Way's conclusion to highlight that late adolescence is also marked by an increased "risk for depression and feelings of isolation" as close childhood friendships become superficial with minimal emotional closeness (p. 60). As boys turn into men, they frequently base their friendships on sports or work rather than emotional connection, resulting in friendships lacking depth and longevity (p. 56).

With the rigid expectations of masculinity, minority boys and Giese's Anishinaabe son 
experience manliness differently than White boys. Sociologist Alexander Lu examines how race and ethnicity perpetuate masculinity (p. 6). Asian boys are often perceived as emasculated by stereotypes like "obedient, nerdy and weak" (p. 6). Additionally, Muslim boys of "South Asian and North African descent" often fall outside of the Man Box with Asian boys and are stereotyped as "model minorities" (p. 7). In contrast to the model minority myth, the adultification of Black boys perceives them as obtaining more traits from the Man Box (p. 81). Adultification results in harsher punishments compared to their non-Black peers (p. 81). The president of Cleveland Police Patrolmen's Association described Tamir Rice as "a 12-year-old boy in an adult body" (p. 81).

In addition to the adultification of Black boys, nearly half the Portuguese boys interviewed by Ph.D. candidate David Pereira defined masculinity in traditional ways, one following strict gender roles (p. 98-99). The emphasis of self reliance as a masculine trait impacts other areas of their life such as school, in which many avoid asking for academic help (p. 99). In Canada, Black and Indigenous boys are punished more severely by school administration than White boys and girls (p. 73). Founder and principal of Mott Hall Bridges Academy Nadia L. Lopez emphasize that boys of colour are assumed to be "out of control" (p. 75). Public education has also been a force of social control, with the history of segregation during the Jim Crow era in the United States and residential schools in Canada (pp. 76-77). Although laws have changed, segregation still exists in public education. Minority boys are more likely to be observed by school administration as having ADHD or ASD (p. 90). They are often labelled "unmanageable" as schools have insufficient counselling and diversion programs to help troubled kids (pp. 86-87). Giese indicates the narrow 
assumption of "the boy crisis" in schools stems from racism and poverty more than the "girlification" of school (pp. 94-95).

Toxic masculinity from the Man Box perpetuates itself into sports and sex. Sports help express masculinity that is "physically fearless and driven to dominate" (p. 118). Lacking interest in sports threatens masculinity; boys embrace playing sports as a part of their identity (p. 112). Sociologist Kathleen E. Miller coined "toxic jock," where students develop their sense of identity through sports (p. 118). The "toxic jock" focuses on the ego-oriented approach of sports participation; they also drink heavily and engage in unprotected sex (p. 119). Toxic jocks are predominately male and embrace labels from the Man Box. Giese introduces Trump's locker-room banter in which he implies that sexually assaulting women is regularly discussed and joked about when men are alone together (p. 125). Hypermasculinity found in sports also affects the understanding of sex; society expects boys to be "sexually aggressive and in charge" (p. 165). The US Department of Justice and the Department of Education reported 4,400 rapes and 2,300 fondling incidences in 2014 (p. 166). Minimal knowledge about consent and the effects of porn on sexual behaviour attributes to the rise in sexual assault cases. Boys often turn to porn for sexual knowledge as many are embarrassed to ask questions during sex education (pp. 168-169). Giese indicates that although boys crave closeness, they struggle to achieve intimacy in relationships because of the expectation to be emotionally invulnerable (p. 183).

Giese's book intends to understand her son's experiences through extensive research and information from educators and statistics. Her academic audience cam consists of anthropologists, sociologists, psychologists, and social workers, as it closely examines information relevant to each 
profession. Additionally, her book can benefit boys themselves because it validates their experiences. Her work covers a variety of research from scholars and educators; however, direct testimonies from boys themselves would enhance the details of her sources. Interviewing her son would add strength to her research and provide personal insight. Giese's book "Boys: What it Means to Become a Man" offers in-depth discussion into the realities of manhood and expectations perpetuated by the restrictions of contemporary masculinity. 